\title{
QUASIMODULAR FORMS AND COHOMOLOGY
}

\author{
MIN HO LEE
}

(Received 7 September 2011)

\begin{abstract}
We construct linear maps from the spaces of quasimodular forms for a discrete subgroup $\Gamma$ of $\operatorname{SL}(2, \mathbb{R})$ to some cohomology spaces of the group $\Gamma$ and prove that these maps are equivariant with respect to appropriate Hecke operator actions. The results are obtained by using the fact that there is a correspondence between quasimodular forms and certain finite sequences of modular forms.
\end{abstract}

2010 Mathematics subject classification: primary 11F11, secondary 11F12, 11F25, 11F75.

Keywords and phrases: quasimodular forms, modular forms, cohomology.

\section{Introduction}

It is well known that there is a Hecke equivariant map from modular forms of a given weight for a discrete subgroup $\Gamma$ of $S L(2, \mathbb{R})$ to cocycles in some cohomology group of $\Gamma$, which determines the Eichler-Shimura isomorphism (see, for example, [3]). The goal of this paper is to study a quasimodular analogue of such a map.

Quasimodular forms generalise modular forms and were introduced by Kaneko and Zagier in [4]. Various arithmetic and geometric aspects of quasimodular forms have been investigated actively in recent years (see, for example, [2, 6, 8, 10, 12]). Given integers $m$ and $\lambda$ with $m \geq 0$, a holomorphic function $f$ on the Poincaré upper halfplane $\mathcal{H}$ is a quasimodular form for $\Gamma$ of weight $\lambda$ and depth at most $m$ if there are holomorphic functions $f_{0}, f_{1}, \ldots, f_{m}$ on $\mathcal{H}$ such that

$$
(c z+d)^{-\lambda} f\left(\frac{a z+b}{c z+d}\right)=f_{0}(z)+\frac{c}{c z+d} f_{1}(z)+\cdots+\left(\frac{c}{c z+d}\right)^{m} f_{m}(z)
$$

for all $z \in \mathcal{H}$ and $\left(\begin{array}{ll}a & b \\ c & d\end{array}\right) \in \Gamma$. It is known that there is a correspondence between quasimodular forms and certain sequences of modular forms (see also [5]). More precisely, a quasimodular form can be expressed as a linear combination of derivatives of the corresponding modular forms.

Hecke operators play an important role in the theory of modular forms or more general automorphic forms, and they can be extended to the case of quasimodular

(C) 2011 Australian Mathematical Publishing Association Inc. 0004-9727/2011 \$16.00 
forms. If $\mathfrak{S}^{n}\left(\mathbb{C}^{2}\right)$ denotes the complex vector space $\mathbb{C}^{n+1}$ equipped with the $\Gamma$-module structure given by the $n$th symmetric tensor representation of $\Gamma$, then a modular form of weight $n+2$ determines a cocycle in the cohomology group $H^{1}\left(\Gamma, \mathbb{S}^{n}\left(\mathbb{C}^{2}\right)\right)$, which can be used to establish the Eichler-Shimura isomorphism. Such a map allows us to introduce Hecke operators on the cohomology space of the form $H^{1}\left(\Gamma, \mathfrak{S}^{n}\left(\mathbb{C}^{2}\right)\right)$ in such a way that the given map is Hecke equivariant. Hecke operators on the cohomology of more general groups were also investigated by Rhie and Whaples in [11].

In this paper we construct linear maps from the spaces of quasimodular forms for a discrete subgroup $\Gamma$ of $S L(2, \mathbb{R})$ to some cohomology spaces of the group $\Gamma$ with coefficients of the form $\mathfrak{S}^{\ell}\left(\mathbb{C}^{2}\right)$ for some nonnegative integer $\ell$ and prove that these maps are equivariant with respect to appropriate Hecke operator actions. The results are obtained by using the above-mentioned correspondence between quasimodular forms and certain finite sequences of modular forms.

\section{Quasimodular forms}

Let $\mathcal{H}$ be the Poincaré upper half-plane on which the group $\operatorname{SL}(2, \mathbb{R})$ acts as usual by linear fractional transformations. Thus we may write

$$
\gamma z=\frac{a z+b}{c z+d}
$$

for all $z \in \mathcal{H}$ and $\gamma=\left(\begin{array}{ll}a & b \\ c & d\end{array}\right) \in S L(2, \mathbb{R})$. For the same $z$ and $\gamma$, we set

$$
\mathfrak{J}(\gamma, z)=c z+d, \quad \Re(\gamma, z)=\frac{c}{c z+d} .
$$

The resulting maps $\mathfrak{J}, \mathfrak{R}: S L(2, \mathbb{R}) \times \mathcal{H} \rightarrow \mathbb{C}$ can be shown to satisfy

$$
\begin{gathered}
\mathfrak{J}\left(\gamma \gamma^{\prime}, z\right)=\mathfrak{I}\left(\gamma, \gamma^{\prime} z\right) \mathfrak{I}\left(\gamma^{\prime}, z\right), \\
\mathfrak{K}\left(\gamma \gamma^{\prime}, z\right)=\mathfrak{J}\left(\gamma^{\prime}, z\right)^{-2} \mathfrak{K}\left(\gamma, \gamma^{\prime} z\right)+\mathfrak{R}\left(\gamma^{\prime}, z\right),
\end{gathered}
$$

for all $\gamma, \gamma^{\prime} \in S L(2, \mathbb{R})$ and $z \in \mathcal{H}$.

Let $R$ be the ring of holomorphic functions $f: \mathcal{H} \rightarrow \mathbb{C}$ satisfying the growth condition

$$
|f(z)| \ll\left(\frac{\mathfrak{J} z}{1+|z|^{2}}\right)^{-v}
$$

for some $v>0$ (see, for example, [7, Section 17.1] for a more precise description of this condition). We fix a nonnegative integer $m$ and denote by $R_{m}[X]$ the complex vector space of polynomials in $X$ over $R$ of degree at most $m$. If a polynomial $\Phi(z, X) \in R_{m}[X]$ is of the form

$$
\Phi(z, X)=\sum_{r=0}^{m} \phi_{r}(z) X^{r}
$$


and $\lambda$ is an integer with $\lambda>2 \mathrm{~m}$, we introduce two additional polynomials

$$
\left(\Xi_{\lambda}^{m} \Phi\right)(z),\left(\Lambda_{\lambda}^{m} \Phi\right)(z) \in R_{m}[X],
$$

defined by

$$
\left(\Xi_{\lambda}^{m} \Phi\right)(z, X)=\sum_{r=0}^{m} \phi_{r}^{\Xi}(z) X^{r}, \quad\left(\Lambda_{\lambda}^{m} \Phi\right)(z, X)=\sum_{r=0}^{m} \phi_{r}^{\Lambda}(z) X^{r},
$$

where

$$
\begin{aligned}
& \phi_{r}^{\Xi}= \frac{1}{r !} \sum_{j=0}^{m-r} \frac{1}{j !(\lambda-2 r-j-1) !} \phi_{m-r-j}^{(j)}, \\
& \phi_{r}^{\Lambda}=(\lambda+2 r-2 m-1) \sum_{j=0}^{r} \frac{(-1)^{j}}{j !}(m-r+j) ! \\
& \times(2 r+\lambda-2 m-j-2) ! \phi_{m-r+j}^{(j)},
\end{aligned}
$$

for each $r \in\{0,1, \ldots, m\}$. These formulas determine isomorphisms

$$
\Lambda_{\lambda}^{m}, \Xi_{\lambda}^{m}: R_{m}[X] \stackrel{\approx}{\rightarrow} R_{m}[X]
$$

with

$$
\left(\Lambda_{\lambda}^{m}\right)^{-1}=\Xi_{\lambda}^{m}
$$

(see [5]).

Given $\gamma \in S L(2, \mathbb{R}), \lambda \in \mathbb{Z}, f \in R$ and $\Phi(z, X) \in R_{m}[X]$ as in (2.4), we set

$$
\begin{gathered}
\left(\left.f\right|_{\lambda} \gamma\right)(z)=\mathfrak{I}(\gamma, z)^{-\lambda} f(\gamma z), \\
\left(\left.\Phi\right|_{\lambda} ^{X} \gamma\right)(z, X)=\sum_{r=0}^{m}\left(\left.\phi_{r}\right|_{\lambda+2 r} \gamma\right)(z) X^{r}, \\
\left(\Phi \|_{\lambda} \gamma\right)(z, X)=\mathfrak{J}(\gamma, z)^{-\lambda} \Phi\left(\gamma z, \mathfrak{J}(\gamma, z)^{2}(X-\mathfrak{H}(\gamma, z))\right)
\end{gathered}
$$

for all $z \in \mathcal{H}$. Using (2.2) and (2.3), it can be shown that the operations $\left.\right|_{\lambda},\left.\right|_{\lambda} ^{X}$ and $\|_{\lambda}$ determine right actions of $S L(2, \mathbb{R})$ on $R$ for the first one and on $R_{m}[X]$ for the other two. Furthermore, the two actions on $R_{m}[X]$ are compatible in such a way that

$$
\begin{gathered}
\left(\left(\Xi_{\lambda}^{m} \Phi\right) \|_{\lambda} \gamma\right)(z, X)=\Xi_{\lambda}^{m}\left(\left.\Phi\right|_{\lambda-2 m} ^{X} \gamma\right)(z, X), \\
\left(\left.\left(\Lambda_{\lambda}^{m} \Phi\right)\right|_{\lambda-2 m} ^{X} \gamma\right)(z, X)=\Lambda_{\lambda}^{m}\left(\Phi \|_{\lambda} \gamma\right)(z, X)
\end{gathered}
$$

for all $\gamma \in S L\left(2, \mathbb{R}\right.$ ), where $\Xi_{\lambda}^{m}$ and $\Lambda_{\lambda}^{m}$ are the isomorphisms in (2.6) (see also [5]).

We now fix a discrete subgroup $\Gamma$ of $\operatorname{SL}(2, \mathbb{R})$ and consider the restrictions of the $S L(2, \mathbb{R})$ actions described above to $\Gamma$.

Definition 2.1. (i) An element $f \in R$ is a modular form for $\Gamma$ of weight $\lambda$ if it satisfies

$$
\left.f\right|_{\lambda} \gamma=f
$$

for all $\gamma \in \Gamma$. 
(ii) A modular polynomial for $\Gamma$ of weight $\lambda$ and degree at most $m$ is an element $F(z, X) \in R_{m}[X]$ satisfying

$$
\left.F\right|_{\lambda} ^{X} \gamma=F
$$

for all $\gamma \in \Gamma$.

(iii) An element $\Phi(z, X) \in R_{m}[X]$ is a quasimodular polynomial for $\Gamma$ of weight $\lambda$ and degree at most $m$ if it satisfies

$$
\Phi \|_{\lambda} \gamma=\Phi
$$

for all $\gamma \in \Gamma$.

We denote by $M_{\lambda}(\Gamma)$ the space of modular forms for $\Gamma$ of weight $\lambda$ and by $M P_{\lambda}^{m}(\Gamma)$ and $Q P_{\lambda}^{m}(\Gamma)$ the spaces of modular polynomials and quasimodular polynomials, respectively, for $\Gamma$ of weight $\lambda$ and degree at most $m$. From (2.6) we see that the maps $\Xi_{\lambda}^{m}$ and $\Lambda_{\lambda}^{m}$ induce the isomorphisms

$$
\Xi_{\lambda}^{m}: M P_{\lambda-2 m}^{m}(\Gamma) \rightarrow Q P_{\lambda}^{m}(\Gamma), \quad \Lambda_{\lambda}^{m}: Q P_{\lambda}^{m}(\Gamma) \rightarrow M P_{\lambda-2 m}^{m}(\Gamma)
$$

for each $\lambda \in \mathbb{Z}$ with $\lambda>2 m$.

Definition 2.2. Given an integer $\lambda$, an element $\phi \in R$ is a quasimodular form for $\Gamma$ of weight $\lambda$ and depth at most $m$ if there are functions $\phi_{0}, \phi_{1}, \ldots, \phi_{m} \in R$ satisfying

$$
\left(\left.\phi\right|_{\lambda} \gamma\right)(z)=\sum_{r=0}^{m} \phi_{r}(z) \mathfrak{H}(\gamma, z)^{r}
$$

for all $z \in \mathcal{H}$ and $\gamma \in \Gamma$, where $\mathcal{R}(\gamma, z)$ is as in (2.1). We denote by $Q M_{\lambda}^{m}(\Gamma)$ the space of quasimodular forms for $\Gamma$ of weight $\lambda$ and depth at most $m$.

Let $\phi \in R$ be a quasimodular form belonging to $Q M_{\lambda}^{m}(\Gamma)$ satisfying (2.11). Then it is known that for $0 \leq k \leq m$ the function $\phi_{k}$ is a quasimodular form belonging to $Q M_{\lambda-2 k}^{m-k}(\Gamma)$ (see, for example, [7]). In particular, since quasimodular forms of depth 0 are modular forms, $\phi_{m}$ is a modular form belonging to $M_{\lambda-2 m}(\Gamma)$. We define the polynomial $\left(Q_{\lambda}^{m} \phi\right)(z, X) \in R_{m}[X]$ associated with $\phi$ by

$$
\left(Q_{\lambda}^{m} \phi\right)(z, X)=\sum_{r=0}^{m} \phi_{r}(z) X^{r} .
$$

Since $\phi$ determines the functions $\phi_{r}$ uniquely, $Q_{\lambda}^{m} \phi$ is well defined. Furthermore, it can be shown that formula (2.12) determines the isomorphism

$$
Q_{\lambda}^{m}: Q M_{\lambda}^{m}(\Gamma) \rightarrow Q P_{\lambda}^{m}(\Gamma)
$$

for each $\lambda \in \mathbb{Z}$ whose inverse is given by

$$
\left(Q_{\lambda}^{m}\right)^{-1}(\Phi(z, X))=\Phi(z, 0)
$$

for $\Phi(z, X) \in Q P_{\lambda}^{m}(\Gamma)$ (see also [1]). For $0 \leq k \leq m$ we define the projection map

$$
\Pi_{k}^{m}: R_{m}[X] \rightarrow R
$$


by setting

$$
\Pi_{k}^{m} \Phi=\phi_{k}
$$

for $\Phi(z, X)$ as in (2.4). Then

$$
\Pi_{k}^{m}\left(M P_{\lambda}^{m}(\Gamma)\right) \subset M_{\lambda+2 k}(\Gamma), \quad \Pi_{k}^{m}\left(Q P_{\lambda}^{m}(\Gamma)\right) \subset Q M_{\lambda-2 k}^{m-k}(\Gamma) ;
$$

hence by restriction we obtain the maps

$$
\Pi_{k}^{m}: M P_{\lambda}^{m}(\Gamma) \rightarrow M_{\lambda+2 k}(\Gamma), \quad \Pi_{k}^{m}: Q P_{\lambda}^{m}(\Gamma) \rightarrow Q M_{\lambda-2 k}^{m-k}(\Gamma) .
$$

\section{Group cohomology}

Given a positive integer $n$, let $\left\{\mathbf{e}_{1}, \ldots, \mathbf{e}_{n+1}\right\}$ be the standard basis for the complex vector space $\mathbb{C}^{n+1}$, whose elements are regarded as column vectors, and set

$$
\left(\begin{array}{l}
z_{1} \\
z_{2}
\end{array}\right)^{n}=\sum_{k=0}^{n} z_{1}^{n-k} z_{2}^{k} \mathbf{e}_{k+1} \in \mathbb{C}^{n+1}
$$

for $\left(\begin{array}{l}z_{1} \\ z_{2}\end{array}\right) \in \mathbb{C}^{2}$. Then the $n$th symmetric tensor power

$$
\rho_{n}: G L(2, \mathbb{C}) \rightarrow G L(n+1, \mathbb{C})
$$

of the standard representation of $G L(2, \mathbb{C})$ on $\mathbb{C}^{2}$ is given by

$$
\rho_{n}(\gamma)\left(\begin{array}{l}
z_{1} \\
z_{2}
\end{array}\right)^{n}=\left(\gamma\left(\begin{array}{l}
z_{1} \\
z_{2}
\end{array}\right)\right)^{n}
$$

for all $\gamma \in G L(2, \mathbb{C})$. We now define the vector-valued function $\mathbf{v}_{n}: \mathcal{H} \rightarrow \mathbb{C}^{n+1}$ on $\mathcal{H}$ by

$$
\mathbf{v}_{n}(z)=\left(\begin{array}{l}
z \\
1
\end{array}\right)^{n}=\sum_{k=0}^{n} z^{n-k} \mathbf{e}_{k+1}=\sum_{k=0}^{n} z^{k} \mathbf{e}_{n-k+1}
$$

for all $z \in \mathcal{H}$. Then, for $\gamma=\left(\begin{array}{ll}a & b \\ c & d\end{array}\right) \in S L(2, \mathbb{R})$,

$$
\rho_{n}(\gamma) \mathbf{v}_{n}(z)=\left(\begin{array}{c}
a z+b \\
c z+d
\end{array}\right)^{n}=(c z+d)^{n} \mathbf{v}_{n}(\gamma z)=\mathfrak{J}(\gamma, z)^{n} \mathbf{v}_{n}(\gamma z)
$$

where $\mathfrak{I}(\gamma, z)$ is as in $(2.2)$.

We denote by $\mathfrak{S}^{n}\left(\mathbb{C}^{2}\right)$ the complex vector space $\mathbb{C}^{n+1}$ equipped with the structure of a $G L(2, \mathbb{C})$ module given by

$$
(\gamma, v) \mapsto(\operatorname{det} \gamma)^{-n / 2} \rho_{n}(\gamma) v
$$

for $\gamma \in G L(2, \mathbb{C})$ and $v \in \mathbb{C}^{n+1}$. If $\Gamma$ is a discrete subgroup of $S L(2, \mathbb{R}) \subset G L(2, \mathbb{C})$ as in Section 2, its first cohomology group with coefficients in $\mathfrak{S}^{n}\left(\mathbb{C}^{2}\right)$ can be described 
as follows. The set $Z^{1}\left(\Gamma, \mathfrak{S}^{n}\left(\mathbb{C}^{2}\right)\right)$ of 1 -cocycles consists of all maps $u: \Gamma \rightarrow \mathbb{C}^{n+1}$ satisfying

$$
u\left(\gamma \gamma^{\prime}\right)=u(\gamma)+\rho_{n}(\gamma) u\left(\gamma^{\prime}\right)
$$

for all $\gamma, \gamma^{\prime} \in \Gamma$. Given an element $v_{0} \in \mathbb{C}^{n+1}$, the set $B^{1}\left(\Gamma, \mathfrak{S}^{n}\left(\mathbb{C}^{2}\right)\right)$ of coboundaries consists of the maps $v: \Gamma \rightarrow \mathbb{C}^{n+1}$ such that

$$
v(\gamma)=\left(\rho_{n}(\gamma)-1\right) v_{0}
$$

for all $\gamma \in \Gamma$, where 1 is the identity map on $\mathbb{C}^{n+1}$. Then the first cohomology group of $\Gamma$ with coefficients in $\mathfrak{S}^{n}\left(\mathbb{C}^{2}\right)$ is given by

$$
H^{1}\left(\Gamma, \mathfrak{\subseteq}^{n}\left(\mathbb{C}^{2}\right)\right)=\frac{Z^{1}\left(\Gamma, \mathfrak{\subseteq}^{n}\left(\mathbb{C}^{2}\right)\right)}{B^{1}\left(\Gamma, \mathfrak{\subseteq}^{n}\left(\mathbb{C}^{2}\right)\right)} .
$$

We now fix a nonnegative integer $m$ and a point $z_{0} \in \mathcal{H}$ and consider a quasimodular form $\phi \in Q M_{2 v}^{m}(\Gamma)$ satisfying (2.11) with $\lambda=2 v$ for some integer $v>m$. If $k$ and $r$ are integers with $0 \leq r \leq k \leq m$, we set

$$
\widehat{\varpi}_{m, \nu}^{k, r}(\phi)(\gamma)=\sum_{\ell=0}^{2(k+v-m-1)} \int_{z_{0}}^{\gamma z_{0}} \phi_{m-k+r}^{(r)}(z) z^{\ell} \mathbf{e}_{2(k+\nu-m)-\ell-1} d z \in \mathbb{C}^{2(k+v-m)-1}
$$

for all $\gamma \in \Gamma$, where $\left\{\mathbf{e}_{1}, \ldots, \mathbf{e}_{2(k+v-m)-1}\right\}$ is the standard basis for $\mathbb{C}^{2(k+\nu-m)-1}$. Note that the integral is independent of the choice of the path $z_{0} \rightarrow \gamma z_{0}$ because the functions $\phi_{k}$ are holomorphic. We now define the map

$$
\mathcal{L}_{m, v}^{k}(\phi): \Gamma \rightarrow \mathbb{C}^{2(k+\nu-m)-1}
$$

associated with $\phi$ by

$$
\mathcal{L}_{m, v}^{k}(\phi)(\gamma)=\sum_{r=0}^{k} \frac{(-1)^{r}}{r !}(2(k+v-m-1)-r) !(m-k+r) ! \widehat{\varpi}_{m, v}^{k, r}(\gamma)
$$

for all $\gamma \in \Gamma$.

Theorem 3.1. The map $\mathcal{L}_{m, v}^{k}(\phi)$ in (3.6) is a cocycle belonging to $Z^{1}\left(\Gamma, \mathfrak{S}^{2(k+v-m)}\left(\mathbb{C}^{2}\right)\right)$, which induces a complex linear map

$$
\mathcal{L}_{m, v}^{k}: Q M_{2 v}^{m}(\Gamma) \rightarrow H^{1}\left(\Gamma, \mathfrak{\Im}^{2(k+v-m-1)}\left(\mathbb{C}^{2}\right)\right)
$$

sending a quasimodular form $\phi(z, X) \in Q M_{2 v}^{m}(\Gamma)$ to the cohomology class of $\mathcal{L}_{m, v}^{k}(\phi)$ in $H^{1}\left(\Gamma, \mathfrak{S}^{2(k+v-m-1)}\left(\mathbb{C}^{2}\right)\right)$.

Proof. Let $\phi \in Q M_{2 v}^{m}(\Gamma)$, so that $Q_{2 v}^{m}(\phi) \in Q P_{2 v}^{m}(\Gamma)$. Then by (2.10) the polynomial $\left(\Lambda_{2 v}^{m} \circ Q_{2 v}^{m}\right)(\phi)$ belongs to $M P_{2(v-m)}^{m}(\Gamma)$; hence, from (2.16),

$$
\left(\Pi_{k}^{m} \circ \Lambda_{2 v}^{m} \circ Q_{2 v}^{m}\right)(\phi) \in M_{2(k+v-m)}(\Gamma)
$$


for $0 \leq k \leq m$. If $\phi$ satisfies (2.11) with $\lambda=2 v$, using (2.5), (2.12) and (2.15),

$$
\begin{aligned}
\left(\Pi_{k}^{m} \circ \Lambda_{2 v}^{m} \circ Q_{2 v}^{m}\right)(\phi)(z)=( & (k+v-m)-1) \\
& \times \sum_{r=0}^{k} \frac{(-1)^{r}}{r !}(2(k+v-m-1)-r) !(m-k+r) ! \phi_{m-k+r}^{(r)} .
\end{aligned}
$$

On the other hand, using (3.1), (3.5) and (3.7),

$$
\begin{aligned}
\mathcal{L}_{m, v}^{k}(\phi)(\gamma)= & \sum_{r=0}^{k} \sum_{\ell=0}^{2(k+v-m-1)} \frac{(-1)^{r}}{r !}(2(k+v-m-1)-r) !(m-k+r) ! \\
& \times \int_{z_{0}}^{\gamma z_{0}} \phi_{m-k+r}^{(r)}(z) z^{\ell} \mathbf{e}_{2(k+v-m)-\ell-1} d z \\
= & \sum_{r=0}^{k} \frac{(-1)^{r}}{r !}(2(k+v-m-1)-r) !(m-k+r) ! \\
& \times \int_{z_{0}}^{\gamma z_{0}} \phi_{m-k+r}^{(r)}(z) \mathbf{v}_{2(k+v-m-1)}(z) d z .
\end{aligned}
$$

From this and (3.9),

$$
\mathcal{L}_{m, v}^{k}(\phi)(\gamma)=\int_{z_{0}}^{\gamma z_{0}} \frac{\left(\Pi_{k}^{m} \circ \Lambda_{2 v}^{m} \circ Q_{2 v}^{m}\right)(\phi)(z)}{2(k+v-m)-1} \mathbf{v}_{2(k+v-m-1)}(z) d z .
$$

To verify the cocycle condition (3.3) for $\mathcal{L}_{m, v}^{k}(\phi)$, we set

$$
f_{k}=\frac{\left(\Pi_{k}^{m} \circ \Lambda_{2 v}^{m} \circ Q_{2 v}^{m}\right)(\phi)}{2(k+v-m)-1} \in M_{2(k+v-m)}(\Gamma) .
$$

Then, for $\gamma, \gamma^{\prime} \in \Gamma$,

$$
\begin{aligned}
\mathcal{L}_{m, v}^{k}(\phi)\left(\gamma \gamma^{\prime}\right) & =\int_{z_{0}}^{\gamma \gamma^{\prime} z_{0}} f_{k}(z) \mathbf{v}_{2(k+\nu-m-1)}(z) d z \\
& =\int_{z_{0}}^{\gamma z_{0}} f_{k}(z) \mathbf{v}_{2(k+v-m-1)}(z) d z+\int_{\gamma z_{0}}^{\gamma \gamma^{\prime} z_{0}} f_{k}(z) \mathbf{v}_{2(k+v-m-1)}(z) d z \\
& =\mathcal{L}_{m, v}^{k}(\phi)(\gamma)+\int_{z_{0}}^{\gamma^{\prime} z_{0}} f_{k}(\gamma z) \mathbf{v}_{2(k+v-m-1)}(\gamma z) d(\gamma z) .
\end{aligned}
$$

However, using (3.2),

$$
\mathbf{v}_{2(k+v-m-1)}(\gamma z)=\mathfrak{J}(\gamma, z)^{-2(k+\nu-m-1)} \rho_{2(k+v-m-1)}(\gamma) \mathbf{v}_{2(k+v-m-1)}(z) .
$$

Using this and the relations

$$
f_{k}(\gamma z)=\mathfrak{J}(\gamma, z)^{2(k+v-m)} f_{k}(z), \quad d(\gamma z)=\mathfrak{J}(\gamma, z)^{-2} d z
$$


we see that

$$
f_{k}(\gamma z) \mathbf{v}_{2(k+v-m-1)}(\gamma z) d(\gamma z)=\rho_{2(k+v-m-1)}(\gamma) f_{k}(z) \mathbf{v}_{2(k+v-m-1)}(z) d(z) ;
$$

hence, from (3.10),

$$
\begin{aligned}
\int_{z_{0}}^{\gamma^{\prime} z_{0}} f_{k}(\gamma z) \mathbf{v}_{2(k+v-m-1)}(\gamma z) d(\gamma z) & =\rho_{2(k+v-m-1)}(\gamma) \int_{z_{0}}^{\gamma^{\prime} z_{0}} f_{k}(z) \mathbf{v}_{2(k+v-m-1)}(z) d(z) \\
& =\rho_{2(k+v-m-1)}(\gamma) \mathcal{L}_{m, v}^{k}(\phi)\left(\gamma^{\prime}\right) .
\end{aligned}
$$

Thus

$$
\mathcal{L}_{m, v}^{k}(\phi)\left(\gamma \gamma^{\prime}\right)=\mathcal{L}_{m, v}^{k}(\phi)(\gamma)+\rho_{2(k+v-m-1)}(\gamma) \mathcal{L}_{m, v}^{k}(\phi)\left(\gamma^{\prime}\right),
$$

and therefore $\mathcal{L}_{m, v}^{k}(\phi)$ satisfies the cocycle condition (3.3).

From Theorem 3.1 we obtain the complex linear map

$$
\bigoplus_{k=0}^{m} \mathcal{L}_{m, v}^{k}: Q M_{2 v}^{m}(\Gamma) \rightarrow \bigoplus_{k=0}^{m} H^{1}\left(\Gamma, \Im^{2(k+v-m-1)}\left(\mathbb{C}^{2}\right)\right)
$$

for each $v>m$.

\section{Hecke operators}

We extend the formulas for $\mathfrak{J}$ and $\mathfrak{I}$ in $(2.1)$ from $S L(2, \mathbb{R})$ to the group $G L^{+}(2, \mathbb{R})$ of $2 \times 2$ real matrices of positive determinant. We also extend the operations $\left.\right|_{\lambda}$ and $\|_{\lambda}$ of $\operatorname{SL}(2, \mathbb{R})$ in $(2.8)$ and $(2.9)$ to those of $G L^{+}(2, \mathbb{R})$ by setting

$$
\begin{gathered}
\left(\left.f\right|_{\lambda} \alpha\right)(z)=(\operatorname{det} \alpha)^{\lambda / 2} \mathfrak{J}(\alpha, z)^{-\lambda} f(\alpha z) \\
\left(F \|_{\lambda} \alpha\right)(z, X)=\operatorname{det}(\alpha)^{\lambda / 2} \mathfrak{J}(\alpha, z)^{-\lambda} \\
\times F\left(\alpha z, \operatorname{det}(\alpha)^{-1} \mathfrak{J}(\alpha, z)^{2}(X-\mathfrak{K}(\alpha, z))\right)
\end{gathered}
$$

for all $z \in \mathcal{H}, \alpha \in G L^{+}(2, \mathbb{R}), f \in R$ and $F(z, X) \in R_{m}[X]$.

Let $\Gamma$ be a discrete subgroup of $\operatorname{SL}(2, \mathbb{R})$ as in Section 2 , and let $\widetilde{\Gamma}$ be its commensurator, that is, the set of elements $g \in G L^{+}(2, \mathbb{R})$ such that $g \Gamma g^{-1} \cap \Gamma$ has finite index in both $\Gamma$ and $g \Gamma g^{-1}$. Given $\alpha \in \widetilde{\Gamma}$, the double coset $\Gamma \alpha \Gamma$ has a decomposition of the form

$$
\Gamma \alpha \Gamma=\coprod_{i=1}^{s} \Gamma \alpha_{i}
$$

for some $\alpha_{i} \in G L^{+}(2, \mathbb{R})$ with $i=1, \ldots, s$. For the same $\alpha$ and an integer $k$, the corresponding Hecke operator

$$
T_{k}(\alpha): M_{k}(\Gamma) \rightarrow M_{k}(\Gamma)
$$

on modular forms is given by

$$
T_{k}(\alpha) f=\sum_{i=1}^{s}\left(\left.f\right|_{k} \alpha_{i}\right)
$$


for $f \in M_{k}(\Gamma)$ (see, for example, [9]). Such operators can be used to introduce the Hecke operator

$$
T_{\lambda}^{M}(\alpha): M P_{\lambda}^{m}(\Gamma) \rightarrow M P_{\lambda}^{m}(\Gamma)
$$

on modular polynomials by

$$
\left(T_{\lambda}^{M}(\alpha) F\right)(z, X)=\sum_{r=0}^{m}\left(T_{\lambda+2 r}(\alpha) \Pi_{r}^{m} F\right)(z) X^{r}
$$

for $F(z, X) \in M P_{\lambda}^{m}(\Gamma)$, where the projection maps $\Pi_{r}^{m}$ are as in (2.14). On the other hand, for quasimodular polynomials the Hecke operator

$$
T_{\lambda}^{P}(\alpha): Q P_{\lambda}^{m}(\Gamma) \rightarrow Q P_{\lambda}^{m}(\Gamma)
$$

is given by

$$
\left(T_{\lambda}^{P}(\alpha) F\right)(z, X)=\sum_{i=1}^{s}\left(F \|_{\lambda} \alpha_{i}\right)(z, X)
$$

for $F(z, X) \in Q P_{\lambda}^{m}(\Gamma)$, where $\|_{\lambda}$ is as in (4.1) (see [1]). Using this and the isomorphism in (2.13), we can also introduce the corresponding Hecke operator

$$
T_{\lambda}^{Q}(\alpha): Q M_{\lambda}^{m}(\Gamma) \rightarrow Q M_{\lambda}^{m}(\Gamma)
$$

on quasimodular forms by setting

$$
T_{\lambda}^{Q}(\alpha) f=\sum_{i=1}^{s}\left(Q_{\lambda}^{m}\right)^{-1}\left(\left(Q_{\lambda}^{m} f\right) \|_{\lambda} \alpha_{i}\right)=\left(\left(Q_{\lambda}^{m}\right)^{-1} \circ T_{\lambda}^{P}(\alpha) \circ Q_{\lambda}^{m}\right) f
$$

for $f \in Q M_{\lambda}^{m}(\Gamma)$, so that

$$
T_{\lambda}^{P}(\alpha) \circ Q_{\lambda}^{m}=Q_{\lambda}^{m} \circ T_{\lambda}^{Q}(\alpha) .
$$

Theorem 4.1. The linear isomorphisms $\Xi_{\lambda}^{m}$ and $\Lambda_{\lambda}^{m}$ in (2.10) satisfy

$$
T_{\lambda}^{P}(\alpha) \circ \Xi_{\lambda}^{m}=\Xi_{\lambda}^{m} \circ T_{\lambda-2 m}^{M}(\alpha), \quad T_{\lambda-2 m}^{M}(\alpha) \circ \Lambda_{\lambda}^{m}=\Lambda_{\lambda}^{m} \circ T_{\lambda}^{P}(\alpha)
$$

for each $\alpha \in \widetilde{\Gamma}$.

Proof. Given a modular polynomial of the form

$$
F(z, X)=\sum_{r=0}^{m} f_{r}(z) X^{r} \in M P_{\lambda}^{m}(\Gamma)
$$

from (2.5) we see that

$$
\left(\Xi_{\lambda}^{m} F\right)(z, X)=\sum_{r=0}^{m} f_{r}^{\Xi}(z) X^{r}
$$


where

$$
f_{r}^{\Xi}=\sum_{j=0}^{m-r} \frac{1}{r ! j !(\lambda-2 r-j-1) !} f_{m-r-j}^{(j)} .
$$

For $\alpha \in \widetilde{\Gamma}$, using (4.1) and (4.5),

$$
\begin{aligned}
\left(\left(T_{\lambda}^{P}(\alpha) \circ \Xi_{\lambda}^{m}\right) F\right)(z, X) \\
=\sum_{i=1}^{s}\left(\operatorname{det} \alpha_{i}\right)^{\lambda / 2} \mathfrak{J}\left(\alpha_{i}, z\right)^{-\lambda} \\
\quad \times\left(\Xi_{\lambda}^{m} F\right)\left(\alpha_{1} z,\left(\operatorname{det} \alpha_{i}\right)^{-1} \mathfrak{J}\left(\alpha_{i}, z\right)^{2}\left(X-\mathfrak{\Re}\left(\alpha_{i}, z\right)\right)\right) \\
=\sum_{i=1}^{s} \sum_{r=0}^{m}\left(\operatorname{det} \alpha_{i}\right)^{\lambda / 2-r} \mathfrak{J}\left(\alpha_{i}, z\right)^{2 r-\lambda}\left(X-\mathfrak{\Re}\left(\alpha_{i}, z\right)\right)^{r} f_{r}^{\Xi}\left(\alpha_{i} z\right) \\
=\sum_{i=1}^{s} \sum_{r=0}^{m} \sum_{k=0}^{r}\left(\operatorname{det} \alpha_{i}\right)^{\lambda / 2-r} \mathfrak{J}\left(\alpha_{i}, z\right)^{2 r-\lambda}\left(\begin{array}{l}
r \\
k
\end{array}\right)\left(-\mathfrak{K}\left(\alpha_{i}, z\right)\right)^{r-k} X^{k} f_{r}^{\Xi}\left(\alpha_{i} z\right) \\
=\sum_{i=1}^{s} \sum_{k=0}^{m} \sum_{r=k}^{m}(-1)^{r-k}\left(\begin{array}{l}
r \\
k
\end{array}\right)\left(\operatorname{det} \alpha_{i}\right)^{\lambda / 2-r} \mathfrak{J}\left(\alpha_{i}, z\right)^{2 r-\lambda} \mathfrak{R}\left(\alpha_{i}, z\right)^{r-k} f_{r}^{\Xi}\left(\alpha_{i} z\right) X^{k} .
\end{aligned}
$$

Hence

$$
\begin{aligned}
\left(\left(T_{\lambda}^{P}(\alpha) \circ \Xi_{\lambda}^{m}\right) F\right)(z, X)= & \sum_{i=1}^{s} \sum_{k=0}^{m} \sum_{r=k}^{m} \sum_{j=0}^{m-r}\left(\begin{array}{l}
r \\
k
\end{array}\right) \frac{\left(\operatorname{det} \alpha_{i}\right)^{\lambda / 2-r}(-1)^{r-k}}{r ! j !(\lambda-2 r-j-1) !} \\
& \times \mathfrak{J}\left(\alpha_{i}, z\right)^{2 r-\lambda} \mathfrak{\Re}\left(\alpha_{i}, z\right)^{r-k} f_{m-r-j}^{(j)}\left(\alpha_{i} z\right) X^{k} \\
= & \sum_{i=1}^{s} \sum_{k=0}^{m} \sum_{r=0}^{m-k} \sum_{j=0}^{m-k-r}\left(\begin{array}{c}
r+k \\
k
\end{array}\right) \frac{\left(\operatorname{det} \alpha_{i}\right)^{\lambda / 2-r-k}(-1)^{r}}{(r+k) ! j !(\lambda-2 r-2 k-j-1) !} \\
& \times \mathfrak{J}\left(\alpha_{i}, z\right)^{2 r+2 k-\lambda} \mathfrak{K}\left(\alpha_{i}, z\right)^{r} f_{m-r-k-j}^{(j)}\left(\alpha_{i} z\right) X^{k}
\end{aligned}
$$

which can be written in the form

$$
\begin{aligned}
\left(\left(T_{\lambda}^{P} \circ \Xi_{\lambda}^{m}\right) F\right)(z, X) \\
=\sum_{i=1}^{s} \sum_{k=0}^{m} \sum_{\ell=0}^{m-k} \sum_{j=0}^{\ell}\left(\begin{array}{c}
\ell-j+k \\
k
\end{array}\right) \frac{(-1)^{\ell-j}\left(\operatorname{det} \alpha_{i}\right)^{\lambda / 2-k-\ell+j}}{(\ell-j+k) ! j !(\lambda+2 k-2 \ell+j-1) !} \\
\quad \times \mathfrak{J}\left(\alpha_{i}, z\right)^{2 k+2 \ell-2 j-\lambda} \Re\left(\alpha_{i}, z\right)^{\ell-j} f_{m-k-\ell}^{(j)}\left(\alpha_{i} z\right) X^{k}
\end{aligned}
$$

by using $\ell=j+r$. On the other hand, from (4.4),

$$
\begin{aligned}
T_{\lambda-2 m}^{M}(\alpha) F(z, X) & =\sum_{k=0}^{m}\left(T_{\lambda+2 k-2 m}(\alpha) f_{k}\right)(z) X^{k} \\
& =\sum_{i=1}^{s} \sum_{k=0}^{m}\left(\left.f_{k}\right|_{\lambda+2 k-2 m} \alpha_{i}\right)(z) X^{k} .
\end{aligned}
$$


Thus, if we set

$$
\phi_{k}=\sum_{i=1}^{s}\left(\left.f_{k}\right|_{\lambda+2 k-2 m} \alpha_{i}\right)
$$

and use (2.5), then

$$
\begin{aligned}
\left(\Xi_{\lambda}^{m} \circ T_{\lambda-2 m}^{M}(\alpha)\right) F(z, X) & =\sum_{k=0}^{m} \sum_{\ell=0}^{m-k} \frac{1}{k ! \ell !(\lambda-2 k-\ell-1) !} \phi_{m-k-\ell}^{(\ell)}(z) X^{k} \\
& =\sum_{i=1}^{s} \sum_{k=0}^{m} \sum_{\ell=0}^{m-k} \frac{1}{k ! \ell !(\lambda-2 k-\ell-1) !} \frac{d^{\ell}}{d z^{\ell}}\left(\left.f_{m-k-\ell}\right|_{\lambda-2 k-2 \ell} \alpha_{i}\right) X^{k} .
\end{aligned}
$$

Note, however, that

$$
\begin{aligned}
\frac{d^{\ell}}{d z^{\ell}}\left(\left.f_{m-k-\ell}\right|_{\lambda-2 k-2 \ell} \alpha_{i}\right)= & \sum_{j=0}^{\ell}(-1)^{\ell-j} \frac{\ell !}{j !}\left(\begin{array}{c}
\lambda-2 k-\ell-1 \\
\ell-j
\end{array}\right)\left(\operatorname{det} \alpha_{i}\right)^{\lambda / 2-\ell+j-k} \\
& \times \mathfrak{J}\left(\alpha_{i}, z\right)^{2 k+2 \ell-2 j-\lambda} \mathfrak{R}\left(\alpha_{i}, z\right)^{\ell-j} f_{m-k-\ell}^{(j)}\left(\alpha_{i} z\right) .
\end{aligned}
$$

Thus

$$
\begin{aligned}
\left(\Xi_{\lambda}^{m} \circ T_{\lambda-2 m}^{M}(\alpha)\right) F(z, X)= & \sum_{i=1}^{s} \sum_{k=0}^{m} \sum_{\ell=0}^{m-k} \sum_{j=0}^{\ell} \frac{(-1)^{\ell-j}\left(\operatorname{det} \alpha_{i}\right)^{\lambda / 2-\ell+j-k}}{k ! j !(\lambda-2 k-\ell-1) !}\left(\begin{array}{c}
\lambda-2 k-\ell-1 \\
\ell-j
\end{array}\right) \\
& \times \mathfrak{J}\left(\alpha_{i}, z\right)^{2 k+2 \ell-2 j-\lambda} \mathfrak{R}\left(\alpha_{i}, z\right)^{\ell-j} f_{m-k-\ell}^{(j)}\left(\alpha_{i} z\right) X^{k},
\end{aligned}
$$

which can easily be shown to coincide with (4.8); hence we obtain the first relation in (4.7). The second relation follows from this and (2.7).

From (4.4), (4.6) and (4.7) we see that the diagram

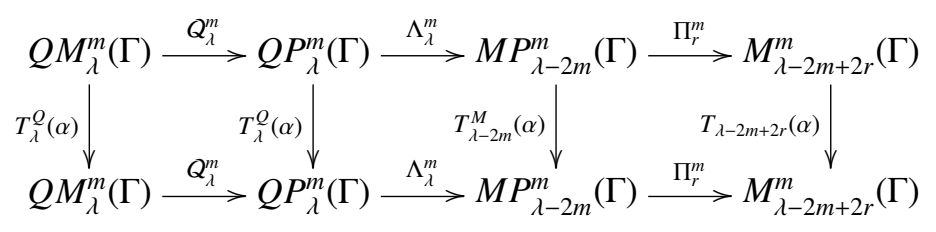

is commutative for each $\alpha \in \widetilde{\Gamma}$ and $r \in\{0,1, \ldots, m\}$.

We now describe Hecke operators on cohomology. Given an element $\alpha \in \widetilde{\Gamma} \subset$ $G L^{+}(2, \mathbb{R})$ such that the corresponding double coset has a decomposition as in (4.2), the corresponding Hecke operator on the cohomology group $H^{1}\left(\Gamma, \mathbb{S}^{n}\left(\mathbb{C}^{2}\right)\right)$ in $(3.4)$ can be determined as follows. If $\gamma \in \Gamma$, since $\Gamma \alpha \Gamma \gamma=\Gamma \alpha \Gamma$, the decomposition in (4.2) can be written as

$$
\Gamma \alpha \Gamma=\coprod_{i=1}^{s} \Gamma \alpha_{i} \gamma
$$


hence, for $1 \leq i \leq s$,

$$
\alpha_{i} \gamma=\xi_{i}(\gamma) \alpha_{i(\gamma)}
$$

for some element $\xi_{i}(\gamma) \in \Gamma$. We note that the set $\left\{\alpha_{1(\gamma)}, \ldots, \alpha_{s(\gamma)}\right\}$ is a permutation of $\left\{\alpha_{1}, \ldots, \alpha_{s}\right\}$. Then the Hecke operator $T_{n}^{H}(\alpha)$ on the cohomology space $H^{1}\left(\Gamma, \mathbb{S}^{n}\left(\mathbb{C}^{2}\right)\right)$ is given by

$$
\left(T_{n}^{H}(\alpha)(\phi)\right)(\gamma)=\sum_{i=1}^{s}\left(\operatorname{det} \alpha_{i}\right)^{n / 2} \rho_{n}\left(\alpha_{i}\right) \phi\left(\xi_{i}(\gamma)\right)
$$

for each 1-cocycle $\phi$ and $\gamma \in \Gamma$ (see [11]).

Theorem 4.2. The linear map $\mathcal{L}_{m, v}^{k}$ with $k, v \in \mathbb{Z}$ and $0 \leq k \leq m<v$ in (3.8) satisfies

$$
\mathcal{L}_{m, v}^{k} \circ T_{2 v}^{Q}=T_{2(k+v-m-1)}^{H}(\alpha) \circ \mathcal{L}_{m, v}^{k}
$$

for each $\alpha \in \widetilde{\Gamma}$.

Proof. Given $\alpha \in \widetilde{\Gamma}$ and $k, v \in \mathbb{Z}$ with $0 \leq k \leq m<v$, from the commutative diagram (4.9) we obtain

$$
T_{2(k+v-m)}(\alpha) \circ \Pi_{k}^{m} \circ \Lambda_{2 v}^{m} \circ Q_{2 v}^{m}=\Pi_{k}^{m} \circ \Lambda_{2 v}^{m} \circ Q_{2 v}^{m} \circ T_{2 v}^{Q}(\alpha) .
$$

Let $\phi \in Q M_{2 v}^{m}(\Gamma)$, and let $f_{k} \in M_{2(k+v-m)}(\Gamma)$ be the associated modular form given by (3.11). Using (3.10), (3.12) and (4.12) and assuming that the double coset $\Gamma \alpha \Gamma$ has a decomposition as in (4.2),

$$
\begin{aligned}
\mathcal{L}_{m, v}^{k} & \left(T_{2 v}^{Q}(\alpha) \phi\right)(\gamma) \\
\quad & =\int_{z_{0}}^{\gamma z_{0}}\left(T_{2(k+v-m)}(\alpha) f_{k}\right)(z) \mathbf{v}_{2(k+v-m-1)}(z) d z \\
& =\sum_{i=1}^{s}\left(\operatorname{det} \alpha_{i}\right)^{k+v-m} \mathfrak{J}\left(\alpha_{i}, z\right)^{-2(k+v-m)} \int_{z_{0}}^{\gamma z_{0}} f_{k}\left(\alpha_{i} z\right) \mathbf{v}_{2(k+v-m-1)}(z) d z \\
& =\sum_{i=1}^{s}\left(\operatorname{det} \alpha_{i}\right)^{k+v-m} \rho_{2(k+v-m-1)}\left(\alpha_{i}\right)^{-1} \int_{z_{0}}^{\gamma z_{0}} f_{k}\left(\alpha_{i} z\right) \mathbf{v}_{2(k+v-m-1)}\left(\alpha_{i} z\right) d\left(\alpha_{i} z\right) \\
& =\sum_{i=1}^{s}\left(\operatorname{det} \alpha_{i}\right)^{k+v-m} \rho_{2(k+v-m-1)}\left(\alpha_{i}\right)^{-1} \int_{\alpha_{i} z_{0}}^{\alpha_{i} z_{0}} f_{k}(z) \mathbf{v}_{2(k+v-m-1)}(z) d z
\end{aligned}
$$

for all $\gamma \in \Gamma$. Since $\alpha_{i} \gamma=\xi_{i}(\gamma) \alpha_{i(\gamma)}$ by (4.10), the above integral can be written in the form

$$
\begin{aligned}
\int_{\alpha_{i} z_{0}}^{\alpha_{i} \gamma z_{0}}= & \int_{z_{0}}^{\xi_{i}(\gamma) \alpha_{i(\gamma) z_{0}}}-\int_{z_{0}}^{\alpha_{i} z_{0}} \\
= & \left(\int_{z_{0}}^{\xi_{i}(\gamma) \alpha_{i(\gamma)} z_{0}}-\rho_{2(k+\gamma-m-1)}\left(\xi_{i}(\gamma)\right) \int_{z_{0}}^{\alpha_{i(\gamma) z_{0}}}\right) \\
& \quad+\left(\rho_{2(k+\gamma-m-1)}\left(\xi_{i}(\gamma)\right) \int_{z_{0}}^{\alpha_{i(\gamma) z_{0}}}-\int_{z_{0}}^{\alpha_{i} z_{0}}\right) .
\end{aligned}
$$


However, using (3.12), (3.13) and (3.14),

$$
\begin{aligned}
& \left(\int_{z_{0}}^{\xi_{i}(\gamma) \alpha_{i(\gamma) z_{0}}}-\rho_{2(k+v-m-1)}\left(\xi_{i}(\gamma)\right) \int_{z_{0}}^{\alpha_{i(\gamma)} z_{0}}\right) f_{k}(z) \mathbf{v}_{2(k+v-m-1)}(z) d z \\
& \quad=\int_{z_{0}}^{\xi_{i}(\gamma) \alpha_{i(\gamma)} z_{0}} f_{k}(z) \mathbf{v}_{2(k+v-m-1)}(z) d z-\int_{z_{0}}^{\alpha_{i(\gamma)} z_{0}} f_{k}\left(\xi_{i}(\gamma) z\right) \mathbf{v}_{2(k+v-m-1)}\left(\xi_{i}(\gamma) z\right) d\left(\xi_{i}(\gamma) z\right) \\
& \quad=\int_{z_{0}}^{\xi_{i}(\gamma) \alpha_{i(\gamma)} z_{0}} f_{k}(z) \mathbf{v}_{2(k+v-m-1)}(z) d z-\int_{\xi_{i}(\gamma) z_{0}}^{\xi_{i}(\gamma) \alpha_{i(\gamma) z_{0}}} f_{k}(z) \mathbf{v}_{2(k+v-m-1)}(z) d z \\
& =\int_{z_{0}}^{\xi_{i}(\gamma) z_{0}} f_{k}(z) \mathbf{v}_{2(k+v-m-1)}(z) d z=\mathcal{L}_{m, \nu}^{k}(\phi)\left(\xi_{i}(\gamma)\right) .
\end{aligned}
$$

On the other hand,

$$
\begin{aligned}
\left(\rho_{2(k+v-m-1)}\left(\xi_{i}(\gamma)\right)\right. & \left.\int_{z_{0}}^{\alpha_{i(\gamma)} z_{0}}-\int_{z_{0}}^{\alpha_{i} z_{0}}\right) f_{k}(z) \mathbf{v}_{2(k+v-m-1)}(z)(z) d z \\
= & \rho_{2(k+\nu-m-1)}\left(\alpha_{i}\right) \rho_{2(k+v-m-1)}(\gamma) \rho_{2(k+v-m)}\left(\alpha_{i(\gamma)}\right)^{-1} \\
& \times \int_{z_{0}}^{\alpha_{i(\gamma)} z_{0}} f_{k}(z) \mathbf{v}_{2(k+\nu-m-1)}(z) d z \\
& -\int_{z_{0}}^{\alpha_{i} z_{0}} f_{k}(z) \mathbf{v}_{2(k+v-m-1)}(z) d z
\end{aligned}
$$

Using the above relations, (4.11) and the fact that $\operatorname{det} \alpha_{i(\gamma)}=\operatorname{det} \alpha_{i}$,

$$
\begin{aligned}
\mathcal{L}_{m, v}^{k}( & \left.T_{2 v}^{Q}(\alpha) \phi\right)(\gamma) \\
= & \sum_{i=1}^{s}\left(\operatorname{det} \alpha_{i}\right)^{k+v-m} \rho_{2(k+v-m-1)}\left(\alpha_{i}\right)^{-1} \mathcal{L}_{m, v}^{k}(\phi)\left(\xi_{i}(\gamma)\right) \\
& +\rho_{2(k+v-m-1)}(\gamma) \sum_{i=1}^{s}\left(\operatorname{det} \alpha_{i(\gamma)}\right)^{k+v-m} \rho_{2(k+v-m-1)}\left(\alpha_{i(\gamma)}\right)^{-1} \\
& \times \int_{z_{0}}^{\alpha_{i(\gamma)} z_{0}} f_{k}(z) \mathbf{v}_{2(k+v-m-1)}(z) d z \\
& -\sum_{i=1}^{s}\left(\operatorname{det} \alpha_{i}\right)^{k+v-m} \rho_{2(k+v-m-1)}\left(\alpha_{i}\right)^{-1} \int_{z_{0}}^{\alpha_{i} z_{0}} f_{k}(z) \mathbf{v}_{2(k+v-m-1)}(z) d z \\
= & \left(T_{2(k+v-m-1)}^{H}(\alpha) \mathcal{L}_{m, v}^{k}(\phi)\right)(\gamma)+\left(\rho_{2(k+v-m-1)}(\gamma)-1\right) u
\end{aligned}
$$

where 1 is the identity map on $\mathbb{C}^{2(k+v-m)+1}$, and

$$
u=\sum_{i=1}^{s}\left(\operatorname{det} \alpha_{i}\right)^{k+\nu-m} \rho_{2(k+\nu-m-1)}\left(\alpha_{i}\right)^{-1} \int_{z_{0}}^{\alpha_{i} z_{0}} f_{k}(z) \mathbf{v}_{2(k+\nu-m-1)}(z) d z .
$$

Hence $\mathcal{L}_{m, v}^{k}\left(T_{2 v}^{Q}(\alpha) \phi\right)$ and $\left(T_{2(k+v-m-1)}^{H}(\alpha) \mathcal{L}_{m, v}^{k}(\phi)\right)$ belong to the same cohomology class, and therefore the proof of the theorem is complete. 
If the Hecke operator $\bigoplus_{k=0}^{m} T_{2(k+v-m-1)}^{H}(\alpha)$ on $\bigoplus_{k=0}^{m} H^{1}\left(\Gamma, \Im^{2(k+\nu-m-1)}\left(\mathbb{C}^{2}\right)\right)$ is defined componentwise, then we see from Theorem 4.2 that it is compatible with the Hecke operator $T_{2 v}^{Q}(\alpha)$ on $Q M_{2 v}^{m}(\Gamma)$ under the map $\bigoplus_{k=0}^{m} \mathcal{L}_{m, v}^{k}$ in (3.15). Thus we obtain the commutative diagram

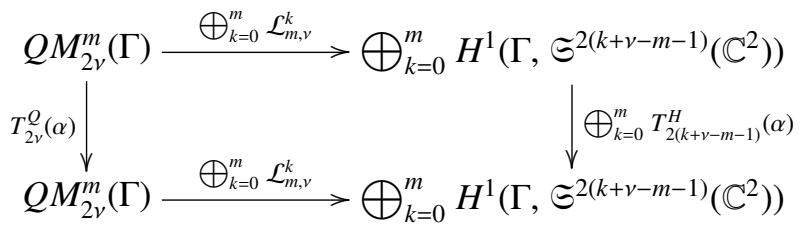

for each $\alpha \in \widetilde{\Gamma}$ and $v>m$.

\section{References}

[1] Y. Choie and M. H. Lee, 'Quasimodular forms, Jacobi-like forms, and pseudodifferential operators', Preprint.

[2] A. Eskin and A. Okounkov, 'Asymptotics of numbers of branched coverings of a torus and volumes of moduli spaces of holomorphic differentials', Invent. Math. 145 (2001), 59-103.

[3] H. Hida, Elementary Theory of L-functions and Eisenstein Series (Cambridge University Press, Cambridge, 1993).

[4] M. Kaneko and D. Zagier, A Generalized Jacobi Theta Function and Quasimodular Forms, Progress in Mathematics, 129 (Birkhäuser, Boston, 1995), pp. 165-172.

[5] M. H. Lee, 'Quasimodular forms and Poincaré series', Acta Arith. 137 (2009), 155-169.

[6] S. Lelièvre and E. Royer, 'Orbitwise countings in $H(2)$ and quasimodular forms', Internat. Math. Res. Not. (2006), 30 Art. ID 42151.

[7] F. Martin and E. Royer, 'Formes modulaires et transcendance', in: Formes Modulaires et Périodes, (eds. S. Fischler, E. Gaudron and S. Khémira) (Soc. Math. de France, 2005), pp. 1-117.

[8] F. Martin and E. Royer, 'Rankin-Cohen brackets on quasimodular forms', J. Ramanujan Math. Soc. 24 (2009), 213-233.

[9] T. Miyake, Modular Forms (Springer, Heidelberg, 1989).

[10] N. Ouled Azaiez, 'The ring of quasimodular forms for a cocompact group', J. Number Theory $\mathbf{1 2 8}$ (2008), 1966-1988.

[11] Y. H. Rhie and G. Whaples, 'Hecke operators in cohomology of groups', J. Math. Soc. Japan 22 (1970), 431-442.

[12] E. Royer, 'Evaluating convolution sums of the divisor function via quasimodular forms', Int. J. Number Theory 21 (2007), 231-262.

MIN HO LEE, Department of Mathematics, University of Northern Iowa, Cedar Falls, IA 50614, USA

e-mail: lee@math.uni.edu 